\title{
Optimization of Speech signal for improving BER using Adaptive 3-D Turbo Codes
}

\section{Suman Kshirsagar}

\begin{abstract}
The introduction of third component in conventional turbo codes improved the code performance for a wide range of block lengths and coding rates with very low error rates. But the parameters such as permeability and permittivity rates were static under noisy environments and hence their adaptability to noisy environment was poor. The proposed $\mathrm{ABD}-\mathrm{TC}$ has overcome the aforesaid problem. The parameters are made adaptive by generating a Genetic Algorithm (GA) based knowledge source. The bit error rate was minimized by generating parameters based on noise and signal strengths. The improvement is observed for speech signal. At high noise rates the speech signal exhibits minimum bit error rate using this GA based knowledge source and for very few iterations they gave error free signal at low values of signal to noise ratio.
\end{abstract}

Key words- A3D-TC, Genetic Algorithm, permeability rate, permittivity rate, $3 D-T C$, bit error rate, signal to noise ratio, iterations.

\section{INTRODUCTION}

Wireless Mobile communication is one of the fastest growing fields in the telecommunication industry. They provide access to the capabilities of the global network at any time, irrespective of the location or mobility of the user. Due to the progress of Internet and cellular communication, it has become more popular. Therefore, in the present and future mobile communication, data transmission has to be done at high bit rates which may be used for many services like video, high quality audio and also many network related applications like Integrated service digital network (ISDN) etc. Ideally, the transfer of information from the source to destination has to be done in such a way that, the quality of the message is preserved.In this regard, communication system plays a vital role which consists of modulation, a noisy medium, and a demodulation scheme [16]. To ensure reliable communication, $\quad E_{b} / N_{o}$ should be maintained at $1.6 \mathrm{~dB}$ irrespective of how powerful an error control code is.So, it is very important for communication systems to have adequate means for the detection and correction of errors in the information received over communication channel[17].The information has to be

Revised Manuscript Received on November 15, 2019

Suman Kshirsagar, Assistant professor in Department of ECE, CBIT, Hyderabad, INDIA. protected from the errors introduced in the communication channel. The information sequence that has to be transmitted may consist of several parts that have different degrees of significance and hence require different levels of protection against noise. This protection is done by a method called coding, where information is disguised by using different codes. Codes are the set of symbols to which meanings or values are attached [3] and are designed to provide different levels of data protection [8]. Hence, the role of error correction codes becomes more prominent.

\section{3-DIMENSIONAL TURBO CODES (3D-TC)}

The introduction of third component in the conventional turbo codes seems to be very effective in improving the code performance. But in these codes the parameters such as permeability and permutation rates seem to be very static and as such these codes cannot perform under different noisy environments. In order to overcome this, the post encoder, permeability rate were involved and permutation was performed. Moreover, the computational complexity remains high in deriving such third component codes. This is found to be the bottleneck in analyzing with 3D EXIT chart analysis and inflexible to q-ary orthogonal modulation. The above shortcomings are resolved by introducing adaptive parameters in the third component. Hence, the code is named as Adaptive Third Component Turbo Code (A3D-TC). The proposed A3D-TC improves the error correction capability by having special intelligence (SI), which decides the permeability and permutation rate of the third component encoder.

\section{III. COMPONENT \\ THE ADAPTIVE THIRD TURBO CODES (A3D-TC)}

In A3D-TC, as shown in fig 1. The third component parameters are made adaptive. This is accomplished by generating a Genetic Algorithm (GA) based knowledge source and feeding it to feed forward neural network. The network outputs third component parameters according to the noise and signal strengths so that bit error rate at decoding section can be minimized in an effective way. The permeability and permutation rates are found with respect to the different noise strength. The data so obtained is utilized to train Artificial Intelligence (AI). 
Optimization of Speech signal for improving BER using Adaptive 3-D Turbo Codes

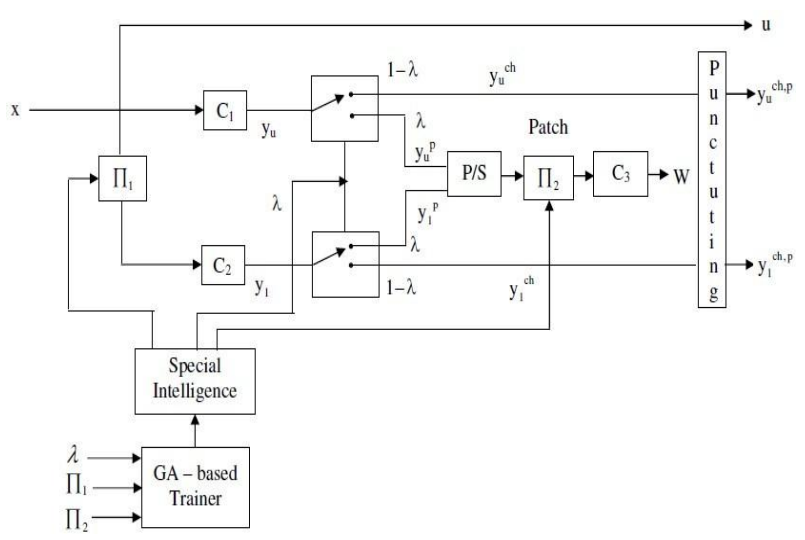

Fig 1: Proposed A3D-TC Encoder

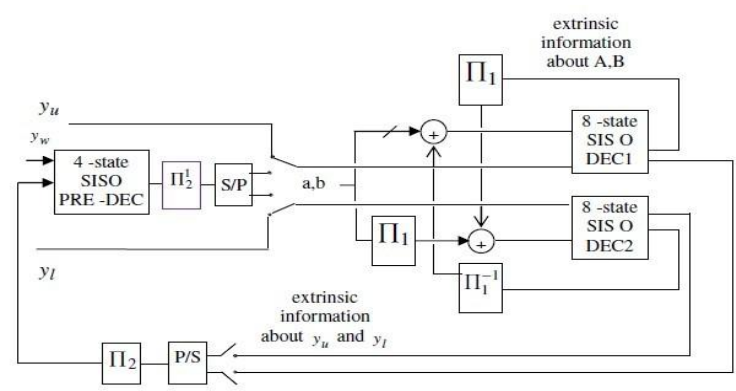

Fig 2: A3D-TC Decoder

The AI technique uses a classifier which will feed forward neural network. The classifier is trained in such a way that the permeability rate and permutation possibilities are decided as per the strength of the noise. As per the decision level, different states of connections are established with post encoder and hence the third component is determined. Such third component will be dynamically varying when the strength of the channel noise varies.By introducing Special Intelligence (SI), A3D- TC improves the error correction capability and decides the permeability and permutation rates of the third component encoder. SI is tuned by generating Genetic Algorithm (GA) based knowledge source and knowledge feeding. Once tuning is completed, the encoder generates third component parameters dynamically according to the noise variance. However the addition of special intelligence in the third component of the encoder never disturbs the conventional third component decoder. The feed forward neural network is used as the special intelligence.

\section{KNOWLEDGE FEEDING}

For the special intelligence appropriate prior knowledge is given which is called as training (or) learning. This is accomplished by generating a precise training dataset, in which noise variance is considered as input for which suitable A3D-TC parameters are generated. The process of prior knowledge feeding includes the generation of knowledge source and then training.

\section{GA-BASED SOURCE}

Given a noise variance, it generates some optimal solutions which are the outputs. For e.g., $\lambda$ The GA based knowledge source is used in ourcoding system to train the special intelligence. This is accomplished by generating a precise training

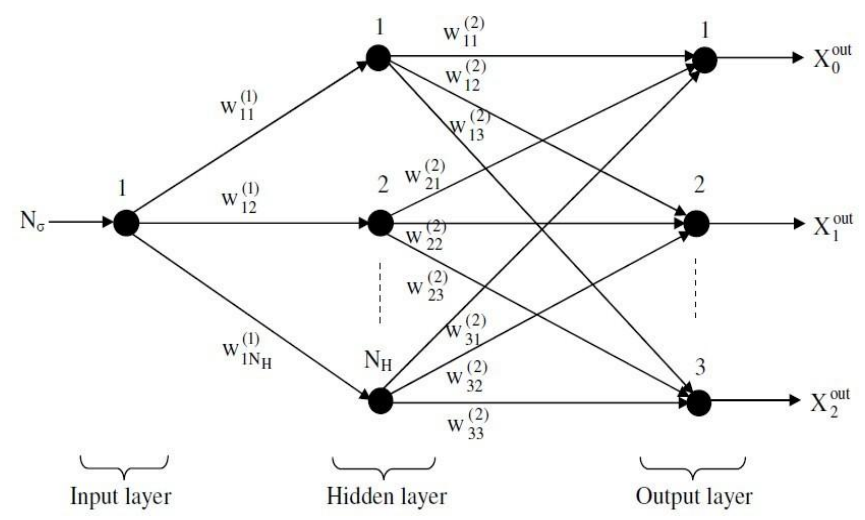

Fig 3: Structure of feed forward neural network used for A3D-TC Encoder

$\Pi_{2}$ are all optimal solutions.data set, in which noise variance is considered as input and the suitable A3D-TC parameters such as permeability rate and permutation rates are generated as outputs. In order to obtain the suitable A3D-TC parameters for the provided noise variance, the classical GA procedure is followed, where a population pool of chromosomes are generated.Each organism in the genetic algorithm has one chromosome. Thus, each chromosome can be thought of as an individual or a solution composed of a collection of parameters to be optimized. These parameters are called genes. Each chromosome is assigned with a random solution or collection of genes. The fitness of every chromosome is evaluated by designing A3D-TC as per the chromosome parameters. It is then encoded with random input bits to which Additive White Gaussian Noise is added to the channel and then data is decoded in the decoder. Hence, the mean bit error rate (BER) is determined.Among all the chromosomes that are present in the population pool, chromosomes with minimum error are selected. Crossover and Mutation is performed, so that genes are exchanged between two chromosomes in crossover operation in order to obtain child chromosomes and the genes are replaced by new genes in every child chromosomes. Single point crossover operation and random mutation is used.

The working of A3D-TC is elaborated as:

Pre analysis is done using Genetic

Algorithm.

i.e., a knowledge source is generated using

Genetic Algorithm

For this knowledge source three parameters $\left(\lambda, \Pi_{1}, \Pi_{2}\right)$ are generated at different noise variances.

Simulation of noise environment is 
done

by GA operation.

This becomes the solution for GA operation.

The main objective is

To minimize the bit error rate the fitness function of GA is found out.

Random chromosomes are generated, i.e., the population pool with around ten chromosomes. These ten chromosomes are selected based on the above three parameters and their corresponding fitness is evaluated for all the ten chromosomes.After crossover and mutation, new chromosomes are formed which combine with the parent chromosomes (selected based on fitness) and form a new population pool. The new population is submitted for fitness evaluation and the process gets repeated till a maximum number of iterations get reached. At the end of the iterations, a best set of A3D-TC parameters are obtained which are used for knowledge feeding for the corresponding noise variance ( $\left.N_{\sigma}\right)$.

\section{Neurons}

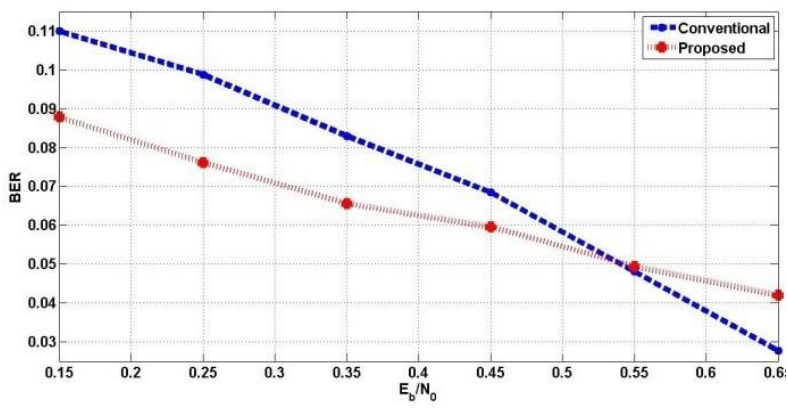

30 Neurons

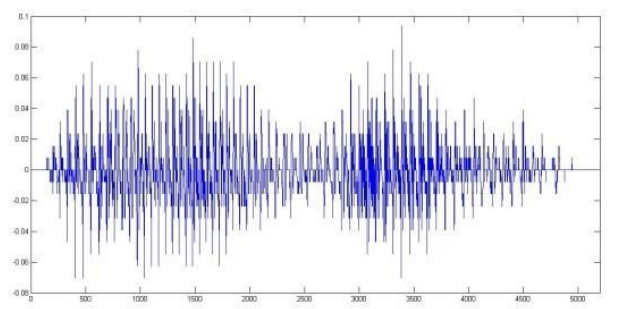

30 Neurons

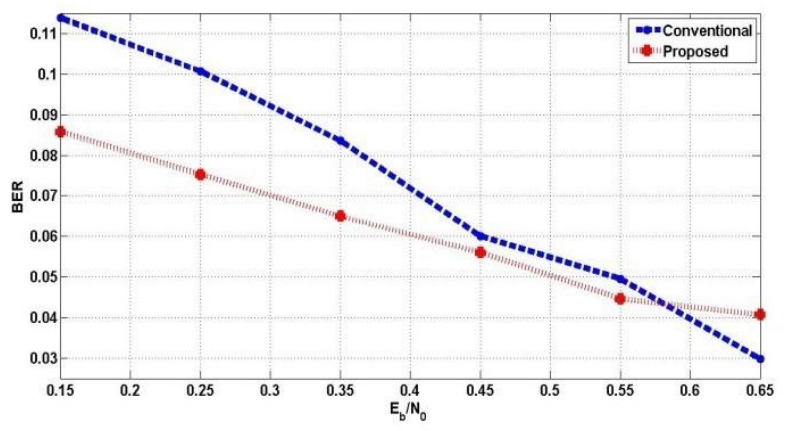

40 Neurons

\section{VI.A3D-TC IN VOICE COMMUNICATION}

The GA based A3D-TC has been compared with the conventional method using speech signal. The speech signal is captured at a sampling frequency of $8 \mathrm{KHz}$ and is converted into a string of bits. These strings of bits are given as input to the algorithm.

\section{VII.RESULTS}

The A3D-TC is implemented and validated in the working platform of MATLAB (version 7.12). The results are shown in the following graphs for 20, 30 and 40 neurons.

\section{Neurons}

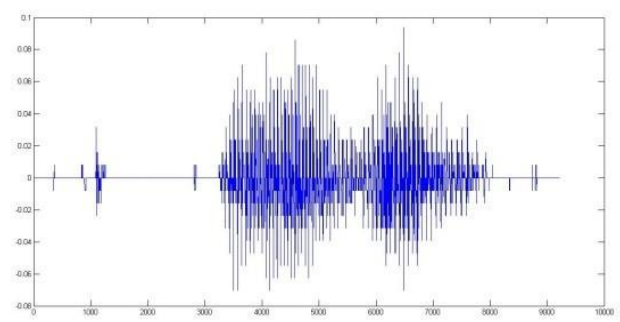

40 Neurons

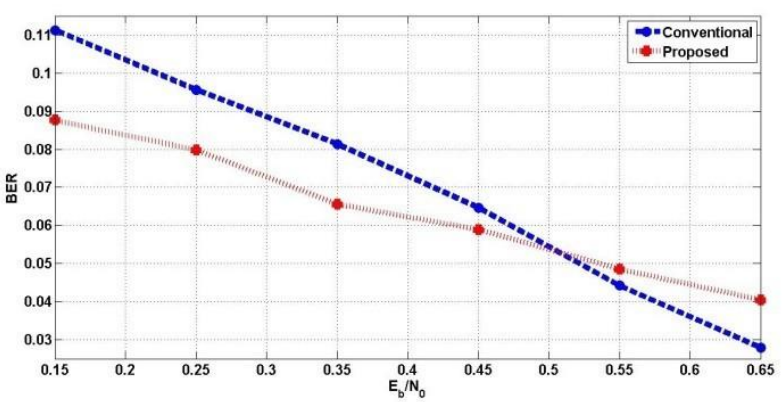

Fig 4: Comparative Chart for BER vs Eb/No performance between A3D-TC (proposed) and 3D- TC (conventional) for network structure with (i) 20, (ii) 30 and (iii) 40 hidden neurons for the speech signal.

It is observed that A3D-TC using Genetic Algorithm exhibits minimum BER when compared with conventional method. For low values of signal to noise ratio, the proposed method using Genetic Algorithm performs better. At high noise rates the speech signal exhibits minimum bit error rate and improved the speech signal.

\section{VIII.CONCLUSION}

In this work, A3D-TC encoder design is developed by including adaptiveness in conventional third component TC. Ten experiments were carried out using both the techniques (conventional and A3D- TC). The influence of neural network structure on the performance of A3D-TC is analyzed by varying the size of the hidden layer. The experimental results show that A3D-TC achieves minimum BER than 3D-TC. It is capable of achieving minimal BER while increasing network complexity.

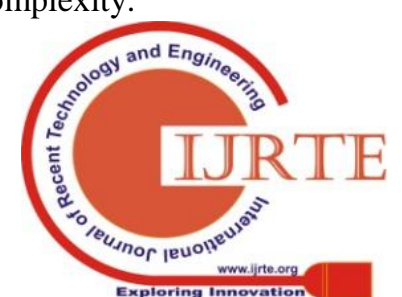


Lesser failure deviation over success deviation.

(ii) It reduces the number of iterations generated, while maintaining the speed.

\section{REFERENCES}

1. Farhan Aadil, Shahzada Khayyam Nisar, Wajahat Abbas and Asim Shahzad, "Reusable IP core for Forward Error Correcting Codes", International Journal of Basic \& Applied Sciences IJBAS-IJENS, Vol.10, No.01, pp.24-30.

2. Joanne Gomes and B K Mishra, "Double Error Correcting Long Code",International Journal of Computer Networks \& Communications (IJCNC), Vol.2,No.5,pp.58 69,2010,DOI10.5121/ijcnc.2010.2504

3. R.W.Hamming, "Error detecting and error correcting codes", The BELL system technical journal, Vol.29, No.2, pp.1-14,1950.

4. Burr A., "Turbo-codes: the ultimate error controlcodes?", Electronics \& Communication EngineeringJournal, Vol.13, No.4,pp. 155 - 165,2001.

5. Said M. Elnoubi, Shawky E. Shabaan and Ahmed H Abd ElMalek, "New Trend in Space Time Error Correcting Codes", Journal of Selected Areas in Telecommunications (JSAT),pp.3239,March Edition, 2011

6. G. A. Lazar,N. R. Buzatu,E. Cojocariu and L. Trifina, "New Deterministic Code Matched Interleaver For Turbo codes", Journal of Theoretical and Applied Information Technology,Vol.10 ,No2.,pp.89-93, 2005

- 2009 .

7. Hamood Shehab and Widad Ismail, "The Development\& Implementation of Reed Solomon Codes for OFDM Using Software-Defined Radio Platform", International Journal of Computer Science \& Communication, Vol. 1, No. 1, pp. 129136,2010

8. Satoshi Yamazaki and David K. Asano, "A SerialUnequal Error Protection Code System Using Multilevel Trellis Coded Modulation with Ring-type Signal Constellations for AWGN Channels", IEEJ transactions on electrical and electronic engineering,Vol. 5,No.6,pp.708716,2010,doi:10.1002/tee.20596

9. Daniel J. Costello, Joachim Hagenauer,Hideki Imai and Stephen B. Wicker, " Applications of Error- Control Coding", IEEE Transactions on Information Theory, Vol. 44, No. 6,pp.25312560,1998

10. Byung K. Yi, Sang G. Kim and Raymond L. Pickholtz,"The Softest Handoff Design Using Iterative Decoding (Turbo Coding)", Journal of communications and networks, Vol.2, No.1, pp.76-84, 2000

11. Sishir Kalita, Parismita Gogoi and Kandarpa KumarSarma, "Convolutional Coding Using Booth Algorithm For Application in Wireless Communication", International Journal of Electronic Signals And Systems, Vol. 2 ,No. 1,pp.37 - 41, 2010

12. Yi Cai, Joel M. Morris, Tülay Adalı and Curtis R.Menyuk, "On Turbo Code Decoder Performance in Optical-Fiber Communication Systems With Dominating ASE Noise", Journal of lightwave technology, Vol. 21, No. 3, pp.727-734,2003

13. Chih-Heng Ke, Rung-Shiang Cheng, Chen-Da Tsaiand Ming-Fong Tsai, "Bandwidth Aggregation with Path Interleaving Forward Error Correction Mechanism for Delay-Sensitive Video Streaming in Wireless Multipath Environments", Tamkang Journal of Science and Engineering, Vol. 13, No. 1, pp.1-9 2010.

14. Aso.M.Raymond and C.Arun, "Design and VLSI Implementation of a High Throughput Turbo Decoder", International Journal of Computer Applications, Vol.22, No.3, pp.33-37,2011

15. Hamood Shehab and Widad Ismail, "HardwareImplementation for Error Correction Using SoftwareDefined Radio Platform", European Journal of Scientific Research, Vol.38, No.2,pp.337-350, 2009.

16. Ojo, F. K. and Ogunti, E. O., "Investigation of Carrier-Phase Offsets in Digital Passband Communication Systems for QPSK GrayEncoding using Constant- Phase and Random-Walk Phase Models", European Journal of Scientific Research,Vol.57,No.3 pp.454465, 2011

17. Syed Mahfuzul Aziz and Minh Duc Pham," Implementation of Low Density Parity Check Decoders
Hence, A3D-TC using Genetic Algorithm can be more preferred than 3D-TC.

using a New High Level Design Methodology",Journal Of Computers, Vol. 5, No. 1, pp.81-90,2010.

18. Berrou, C., Graell i Amat, A., Ould-Cheikh-Mouhamedou, Y. and Saouter, Y., "Improving the Distance Properties of Turbo Codes Using a Third Component Code: 3D Turbo Codes", IEEE Transactions on Communications, Vol.57, No.9, pp.2505 - 2509, 2009.

19. Babich, F., Crismani, A. and Maunder, R. G.," EXITChart Aided Design of Periodically Punctured TurboCodes", IEEE Electronics Letters, Vol. 46, No.14, 2010

20. Yongsang Kim, Kyungwhoon Cheun, Kyeongcheol Yang and Min Sagong, "Design of Turbo Codes over GF(q) with q-ary Orthogonal Modulation", IEEE Transactions on Communications, Vol.59, No.3,pp.625

631,2011

21. C.Berrou, A.Graell I.Amat, Y.Ould Cheikh Mouhamedou, C. Douillard, Y. Saouter, "Adding a Rate-1 Third Dimension to Turbo Codes", IEEE Information Theory Workshop, Pages: 156-161, 2007

22. Suman Kshirsagar and Dr. E.Nagabhooshanam, "The A3D-TC: An adaptive Approach for selecting third component parameters to generate Robust Turbo Codes," International Journal of Computer Applications, vol. 52-No.21, August 2012.

\section{AUTHORS PROFILE}

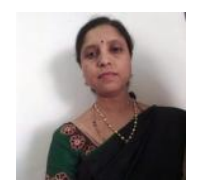

Author is Dr.Suman Kshirsagar working as Assistant professor in Department of ECE, CBIT, Hyderabad, INDIA. She has a doctorate from JNTU Hyderabad. She is specialized in Error Correcting Codes, Electromagnetics, Antennas and Microwaves. She has 15 journals and presented papers in conferences in the above subjects. 